\title{
DOUBLE STARS WITH COMMON PROPER MOTION
}

\author{
WILLEM J. LUYTEN \\ Dept. of Astronomy, University of Minnesota; Minneapolis 14, U.S.A.
}

The proper Motion Survey with the 48-in Schmidt telescope has now progressed far enough such that we may begin to make some meaningful statistical discussions of the results obtained. Altogether 135 regions have been blinked - one seventh of the total and in particular all fifty plates covering the area of the North Polar Cap, north of declination +69 (1855) have been blinked and completely measured. This area covers 1360 square degrees, almost exactly one thirtieth of the entire sphere, and, since the galactic latitude of the Pole is $27^{\circ}$, and its distance from the 'average' soiar apex is about $55^{\circ}$ this region may be considered as not too unrepresentative for the whole sky.

Within this area were found 561 double and triple stars with common proper motion - these are being published in a general catalogue of such doubles which brings the total of LDS objects to well over 2000. About 1000 more are now being measured and will be published shortly. Among the 561 pairs north of $+69^{\circ}$ there are 15 with $\mu \geqslant 0$. $3, m_{B} \leqslant 19^{m} .0$, and with a separation $s \geqslant 6^{\prime \prime}$. Hence, assuming a constant star density we would expect 405 similar pairs with $\mu \geqslant 0.1, m_{B} \leqslant 21^{m} .5$ (the plate limit), and $s \geqslant 2$ ", whereas only 343 were found. This gives an indication of the incompleteness of the survey for the smaller motions. Extrapolating over the whole sky and for motions down to 0.05 (which means stars statistically nearer than 200 parsec) we then find an expected total of about 100000 but since now this would include separations down to $1^{\prime \prime}$ and faint companions down to $m=23 \mathrm{pg}$ the actual number observable would probably be no more than 80000 . Even so, this number exceeds by a wide margin the total number of visually discovered double stars nearer than 200 parsec.

It is evident, therefore, that these relatively wide, common proper motion pairs constitute, observationally speaking, the most common type of binaries in space. Nevertheless, double-star observers and theoreticians alike continue to ignore them in favor of, especially, the exceptionally rare binaries of the OB class. Perhaps this is part of the reason we have progressed so little in our attempts at understanding the processes of double star genesis: we have concentrated our efforts on the exceptional freaks, and have neglected the common man in space.

Perhaps the most interesting of these common-proper-motion pairs are those which contain a white dwarf or degenerate component. These now number 120 , from among more than 2000 LDS pairs. A complete list of the data as well as a preliminary discussion is being published at Minnesota hence $I$ will here only touch on the highlights.

Sixty percent of these pairs appear to be similar to $o_{2}$ Eridani BC - one star has the same color as $\mathrm{O}_{2}$ Eri $\mathrm{B}$, the other that of a late $K$ or $M$. For 39 of them the red component, and for 31 the white component is the fainter, photographically. But when we apply differential color corrections to obtain visual magnitudes, these numbers change to leave only twenty percent with the white component brighter, while, when applying 
further corrections to obtain bolometric magnitudes only four stars have the white component as the brighter. But for three of these the difference in magnitude is only $0^{m} .1$ or $0^{m} \cdot 2$ - much less than the uncertainty in the bolometric corrections, thus leaving only one pair where the white dwarf appears to be bolometrically brighter than the main-sequence star, thus substantiating what I found fifteen years ago from a much smaller sample.

For virtually none of these stars parallaxes are known, and the only indication of luminosity we have is the reduced proper motion $H=m+5+5 \log \mu$, which, for stars found in proper motion surveys roughly averages 6 units larger than the absolute magnitude $M$. If we plotted the data for these stars the dots for all the $o_{2}$ Eri BC type of doubles would cluster very closely around two points in a revised $H$ - $R$, i.e. a diagram plotting $H$ against color, hence it seems more sensible to plot merely the average of them all. For this we find:

$$
\begin{array}{ll}
\text { For the white components } & \bar{H}=17^{\mathrm{m}} .5 \mathrm{pg} \pm 1^{m} .5 \\
\text { For the red components } & \bar{H}=17.5 \mathrm{pg} \pm 2.3
\end{array}
$$

The dispersions are made up of at least four contributing causes viz. the observational errors in the proper motions and the magnitudes, and the cosmic dispersions in the tangential velocities and in the luminosities. Since the proper motions are fairly large we may perhaps provisionally neglect the first of these; the second one is estimated to be around \pm 0.7 . If then we assume, rather arbitrarily, that the third and fourth are equally large for the white stars, we obtain a value of $\pm 0^{m} .9$ for each, indicating a dispersion in $T$ as between 0.7 and 1.5 times the (geometric) mean value. The dispersion in luminosity for the red stars is then found to be $\pm 2^{m} .0$ - which was to be expected, since the main sequence here is much steeper than the white dwarf sequence.

Next we come to 34 doubles were either the degenerate component is later than color class $f$, or the main sequence component earlier than $k$, or both. When these are plotted in a similar diagram we find that for thirty of them the one component lies either on, or below the line which was established in the North Polar Cap as the dividing line between subdwarfs and degenerates. In two more cases the supposedly degenerate component lies about 2 magnitudes above this line - but so does the primary lie well above the main sequence and these may well be pairs with smaller than usual tangential velocities. The remaining two could possibly contain a subdwarf component rather than a genuine degenerate. One of the most interesting pairs is $\xi$ Draconis. If here we accept the trigonometric parallax of 0.031 the primary becomes $\mathrm{K} 0, M=+2.0 \mathrm{pg}-$ quite normal for a $\mathrm{K}$ giant and the secondary has $M=+13.0 \mathrm{pg}$, color $k$, and thus could well be a genuine degenerate.

Finally we have fifteen pairs where both components could be degenerate. The best of them is still LDS 275 , the first double white dwarf discovered. The most noticeable feature of these stars is that for fourteen of them the white component is the brighter one - which is exactly what one would expect if a single white-dwarf sequence exists. In four of these pairs the fainter component has the color of an M star. Obviously, from our crude estimates of color, it is not possible to distinguish between, say, an 
M2 or an M7, so these fainter components could perhaps be extremely late M mainsequence stars. But - if degenerate stars with color $m$ really exist these four would seem to be the most promising candidates.

\section{Discussion}

Heintz: Does the magnitude dispersion for degenerate components still leave the $\mathrm{U}$ Gem type stars as a separate group outside the range? This may be important for the assumption of a rapid evolutionary process.

Luyten: The observed distribution might be due to difference in evolutionary speed, or it might be simply an observational selection: $o_{2}$ Eri B type white dwarfs are easy to find.

Scarfe: You plotted several stars as lying between the main and the white dwarf sequences. Does this imply that the corresponding region in the HR diagram is populated with stars?

Luyten: No, there is a scatter around both the main and the white-dwarf sequences but a relatively empty region between them.

Scarfe: Among single degenerate stars, is it true that the average bolometric magnitude is brighter for red stars than for white ones, as you found for most of the binaries you observed? Or are the available parallaxes insufficient to decide this?

Luyten: I don't think it is true for single stars because you don't know which red star to compare with which degenerate. Even in the average, it depends largely on observational uncertainty, and just what on the average you call a single white dwarf and a single red star.

Further commenting on the objects observed, Luyten says that there is only one case where the red star seems to be definitely the fainter one. In two other cases the red component is 0.1 magnitude brighter than the white one but this is much less than the observational uncertainty in the bolometric correction. Concerning the spectra of degenerate components in spectroscopic binaries, Luyten replies to Asimov that there is no data. In cases such as Castor Aa and Bb where the secondary has been suspected of being a white dwarf there is no chance of observing its spectrum. 